\title{
Complete Arcs and Surfaces in Three Dimensional Projective Space $P G(3,7)$
}

\author{
Ali Ahmed A. Abdulla1, Nada Yassen Kasm Yahya ${ }^{2}$ \\ ${ }^{1}$ Department of Mathematics, College of Computer Science and Mathematics, University of Mosul, Mosul, Iraq \\ ${ }^{2}$ Department of Mathematics, College of Education for Pure Science, University of Mosul, Mosul, Iraq \\ Email: drnadaqasim1@gmail.com
}

How to cite this paper: Abdulla, A.A.A. and Yahya, N.Y.K. (2020) Complete Arcs and Surfaces in Three Dimensional Projective Space $P G(3,7)$. Open Access Library Journal, 7: e6071.

https://doi.org/10.4236/oalib.1106071

Received: January 13, 2020

Accepted: April 23, 2020

Published: April 26, 2020

Copyright $\odot 2020$ by author(s) and Open Access Library Inc.

This work is licensed under the Creative Commons Attribution International License (CC BY 4.0).

http://creativecommons.org/licenses/by/4.0/

\begin{abstract}
The purpose of this thesis is to construct surfaces and complete arcs in the projective 3-space $P G(3, q)$ over Galois fields $G F(q), q=7 . \mathrm{A}(k, n)$-arc in $P G(3, q)$ is a set of $k$ points; no $n+1$ of them are coplanar. $\mathrm{A}(k, n)$-arc is complete if it is not contained in a $(k+1, n)$-arc. In this work the $(k, q)$-span are constructed in $P G(3,7)$ and it is found that, it exists in $P G(3,7)$ when $k=50$. Moreover, the maximum ( $k$, $)$-span, is called a spread.
\end{abstract}

\section{Subject Areas}

Projective Geometry

\section{Keywords}

Algebraic Curves and Surfaces, Complete Arcs and Surfaces in Three Dimensional Projective Space $P G(3, q),(k, 9)-$ Span, Spread, Sets of Subspaces

\section{Introduction}

The study of finite projective spaces was at one time no more than an adjunct to algebraic geometry over the real and complex numbers. But, more recently, finite spaces have been studied both for their application to practical topics such as coding theory and design experiments, and for their illumination of more abstract mathematical topics such as finite group theory and graph theory. Perhaps the fastest growing area of modern mathematics is combinatorics that is concerned with the study of arrangement of elements into sets. These elements are usually finite in number, and the arrangement is restricted by certain boundary conditions imposed by the particular problem under investigation. Much of the 
growth of combinatory has gone hand in hand with the development of the computer. A major reason for this rapid growth of combinatorics is its wealth of applications, to computer science, communications, transportations, genetics, experimental design, and so on.

A recurring theme of this work is the characterization of algebraic varieties in $P G(n, q)$ as finite sets of points with certain combinatorial properties. Among these finite sets, the concept of $(k, n)$-arcs is in the $n$-dimensional projective space over Galois field $G F(q), q=p^{m}$ for some prime number $p$ and some integer $m$.

Hirschfeld, J.W.P. (1979) studies the basic definition and theorems of projective geometrics over finite fields [1], Kareem view ( $k$, $)$-span in $P G(3, p)$ over Galois field $G F(p), p=4$ in 2013 [2]. Al-Mokhtar studies the complete arcs and surface in three-dimensional projective space over Galois field $G F(P), p=2,3$ [3]. In three-dimensional projective space, the control problem is how to construct and find the whole space spread which is (50,0)-span in $P G(3,7)$ and prove it in general when $P \geq 2$ is $p^{2}+1$. Complete arcs have important connections with a number of other objects, see [4]-[19] and the references therein. Hirschfeld, J.W.P. (1998) studies the basic definition and theorems of projective geometrics over finite fields, in 2008 [20]. This paper includes three sections. First section considers the preliminaries of projective 3-space which contains some definition and theorems for the concept, whereas the second section consists of the subspace in $P G(3, p)$. Finally, the third section constructs maximum complete $(k, q)$. A span in $P G(3,7)$ is spreading, and in general proves Geometric rule in (Conclusions) $P \geq 2$. The total number of $(k$, , $)$-span in $P G(3, q)$ is $p^{2}+1, p \geq 2$.

\section{2. ( $k$, $)$-Spread in $P G(3, p)$}

\subsection{Sets of Subspaces}

\section{Qualifier [19]}

A $(k, Q)$-set in $P G(n, q)$ is a set of $k$ spaces $\pi$. A $k$-set is a $(k, 0)$-set, that is a set of $k$ points.

\section{Definition [19]}

A $(k, q ; r, s, n, q)$-set is a $(k, q)$-set in $P G(n, q)$ at most $r$ spaces $\pi_{q}$ of which lie in any $\pi s$.

It is of great interest for applications to find maximal and particularly maximum such sets as $k$ varies but the other parameters remain fixed. Thus a complete $(k, q ; r, s ; n, q)$-set is one not contained in any $(k+1, q ; r, s, n, q)$-set.

The definition of $(k,\{; r, s, n, q)$-set is specialized as follows.

$\mathrm{A}(k, r, s ; n, q)$-set is a $(k, 0 ; r, s, n, q)$-set;

$\mathrm{A}(k, r, n, q)$-set is a $(k, r, r-1 ; n, q)$-set;

A $(k, r)$-cap is a $(\mathrm{k} ; \mathrm{r}, q ; \mathrm{n}, \mathrm{q})$-set with $n \geq 3$;

A $k$-cap is a $(k, 2)$-cap;

A $k$-arc is a $(k, n, n-1 ; n, q)$-set; 
A plane $(k, \mathrm{r})$-arc is a $(k, r, 1 ; 2, q)$-set;

A $(k$, ,)-span is a $(k, 1 ; 1,2$ q; $n, q)$-set with $q \geq 1$.

Thus a plane $k$-arc (or just $k$-arc) is a set of $k$ points in $P G(2, q)$, no three of which are collinear, a maximum plane $k$-arc is an oval.

A $k$-cap is a set of $k$ points in $P G(n, q)$ with $n \geq 3$ such that no three are collinear.

A maximum $k$-cap in $P G(3, q)$ is an ovaloid, denoted by $m(n, q)$.

A $\left(k\right.$, , )-span is a set of $k$ spaces $\pi_{1}$ no two of which intersect, a maximum $(k, 9)$-span is a sprea

\section{Definition}

In $P G(3, q)$, if $K$ is any $k$-set, then an $n$-secant of $K$ is a line (a plane) q such that $|\ell \cap K|=n$. In particular, the following terminology are also used

0 -secant is an external line (plane).

1 -secant is a unisecant line (plane).

2 -secant is a bisecant line (plane).

3-secant is a trisecant line (plane).

\subsection{Algebraic Curves and Surfaces}

\section{Qualifier [6] [7]}

A polynomial $\mathrm{F}$ in a polynomial ring $K\left[x_{1}, \cdots, x_{n}\right]$ is called homogenous or a form of degree $d$ if all of its terms have the same degree $d$.

Qualifier [6] [7]

A subset $v$ of $P G(n, K)$ is a variety (over $K$ ) if there exist forms $F_{1}, F_{2}, \cdots, F_{r}$ in $K\left[x_{1}, \cdots, x_{n}\right]$, such that:

$$
\begin{aligned}
v & =\left\{P(A) \text { in } P G(n, K) \mid F_{1}(A)=F_{2}(A)=\cdots=F_{r}(A)=0\right\} \\
& =V_{n, K}\left(F_{1}, F_{2}, \cdots, F_{r}\right)
\end{aligned}
$$

The points $P(A)$ are points of $v$. When $K=G F(q)$, the notation $V_{n, q}\left(F_{1}, F_{2}, \cdots, F_{r}\right)$ is used.

A variety $V_{n, K}(F)$ in $P G(n, K)$ is a primal. A primal in $P G(2, K)$ is a plane algebraic curve, a primal in $P G(3, K)$ is a surface. The dimension of a primal is $n-1$; in particular, a curve in $P G(2, K)$ and a surface in $P G(3, K)$ have dimensions one and two respectively.

\section{Definition}

A point $N$ not on a $(k, n)$-set $A$ has index $i$ if there are exactly $i$ (n-secants) of $K$ through $N$, one can denote the number of points $N$ of indexi by $C_{i}$.

It is concluded that the $(k, n)$-set is complete iff $C_{0}=0$. Thus the $k$-set is complete iff every point of $P G(3, q)$ lies on some $n$-secant of the $(k, n)$-set.

\section{Qualifier}

$\mathrm{A}(k, n)-\operatorname{arc} A$ in $P G(3, q)$ is a set of $k$ points such that at most $n$ points of which lie in any plane, $n \geq 3$. $n$ is called the degree of the $(k, n)$-arc.

\section{Definition [19]}

Let $T_{i}$ be the total number of the $i$-secants of a $(k, n)$-arc $A$, then the type of $A$ w.r.t. its planes denoted by $\left(T_{n}, T_{n-1}, \cdots, T_{0}\right)$. 


\section{Qualifier [19]}

Let $\left(k_{1}, n\right)$-arc $A$ is of type $\left(T_{n}, T_{n-1}, \cdots, T_{0}\right)$ and $\left(k_{2}, n\right)$-arc $B$ is of type $\left(S_{n}, S_{n-1}, \cdots, S_{0}\right)$, then $A$ and $B$ have the same type iff $T_{i}=S_{i}$, for all $i$, in this case, they are projectively equivalent.

\section{Notion}

Let $t(P)$ represents the number of unisecants (planes) through a point $P$ of a $(k, n)-\operatorname{arc} A$ and let $T_{i}$ represent the numbers of $i$-secants (planes) for the $\operatorname{arc} A$ in $P G(3, q)$, then:

1)

$$
t=t(P)=q^{2}+q+2-k-\frac{(k-1)(k-2)}{2}-\cdots
$$

$$
-\frac{(k-1)(k-2) \cdots(k-(n-1))}{(n-1) !}
$$

2) $T_{1}=k t$

3) $T_{2}=\frac{k(k-1)}{2}$

4) $T_{3}=\frac{k(k-1)(k-2)}{3 !}$

5) $T_{n}=\frac{k(k-1) \cdots(k-n+1)}{n !}$

6)

$$
\begin{aligned}
T_{0}= & q^{3}+q^{2}+q+1-k t-\frac{k(k-1)}{2}-\frac{k(k-1)(k-2)}{3 !}-\cdots \\
& -\frac{k(k-1)(k-2) \cdots(k-n+1)}{n !}
\end{aligned}
$$

\section{Proof}

1) there exist $k-1$ bisecants to $A$ through $P$ and there exist $\left(\begin{array}{c}k-1 \\ 2\end{array}\right)$ trisecants to $A$ through $P$, and so there exist $\left(\begin{array}{l}k-1 \\ n-1\end{array}\right) n$-secants to $A$ through $P$, and since there exist exactly $q^{2}+q+1$ planes through $P$, then the number of the unisecants through $P$.

$$
\begin{aligned}
t(P) & =q^{2}+q+1-(k-1)-\left(\begin{array}{c}
k-1 \\
2
\end{array}\right)-\cdots-\left(\begin{array}{c}
k-1 \\
n-1
\end{array}\right) \\
& =q^{2}+q+2-k-\frac{(k-1)(k-2)}{2}-\cdots-\frac{(k-1)(k-2) \cdots(k-n+1)}{(n-1) !} \\
& =t
\end{aligned}
$$

2) $T_{1}=$ the number of unisecants to $A$, since each point of $A$ has $t$ unisecants and the number of the points of $A$ is $k$, then $T_{1}=k t$.

3) $T_{2}=$ the number of bisecants to $A$, which is the number of planes passing through any two points of $A$. Hence $T_{2}=\left(\begin{array}{l}k \\ 2\end{array}\right)=\frac{k(k-1)}{2}$.

4) $T_{3}=$ the number of trisecants of $A$, which is the number of planes passing through any three points of $A$. Hence $T_{3}=\left(\begin{array}{l}k \\ 3\end{array}\right)=\frac{k(k-1)(k-2)}{3 !}$. 
5) $T_{n}=$ the number of $n$-secants planes to $A$,

$T_{n}=\left(\begin{array}{l}k \\ n\end{array}\right)=\frac{k(k-1) \cdots(k-n+1)}{n !}$

6) $q^{3}+q^{2}+q+1$ represents the number of all planes, then in a $(k, n)$-arc of $P G(3, q), q^{3}+q^{2}+q+1=T_{0}+T_{1}+T_{2}+T_{3}+\cdots+T_{n}$

$$
\begin{aligned}
T_{0}= & q^{3}+q^{2}+q+1-T_{1}-T_{2}-T_{3}-\cdots-T_{n} \\
= & q^{3}+q^{2}+q+1-k t-\frac{k(k-1)}{2}-\frac{k(k-1)(k-2)}{3 !}-\cdots \\
& -\frac{k(k-1)(k-2) \cdots(k-n+1)}{n !}
\end{aligned}
$$

\section{Theorem}

Let $T_{i}$ represent the total number of the $i$-secants for a $(k, n)$-arc $A$ in $P G(3, q)$, then the following equations are satisfied:

1) $\sum_{i=0}^{n} T_{i}=q^{3}+q^{2}+q+1$

2) $\sum_{i=1}^{n} i ! T_{i}=k t+k(k-1)+k(k-1)(k-2)+\cdots+k(k-1)(k-n)$

3) $\sum_{i=2}^{n} i(i-1) T_{i}=k(k-1)+k(k-1)(k-2)+1 / 2 k(k-1)(k-2)(k-3)$

$$
+\cdots+1 /((n-2) !) k(k-1)(k-n)
$$

\section{Proof}

1) $\sum_{i=0}^{n} T_{i}$ represents the sum of numbers of all $i$-secants to $A$, which is the number of all planes in the space. Hence $\sum_{i=0}^{n} T_{i}=q^{3}+q^{2}+q+1$.

2) $T_{1}=k t, t=q^{2}+q+2-k-\frac{(k-1)(k-2)}{2}-\cdots-\frac{(k-1) \cdots(k-n+1)}{(n-1) !}$,

$T_{2}=\frac{k(k-1)}{2}, T_{3}=\frac{k(k-1)(k-2)}{3 !}, T_{4}=\frac{k(k-1)(k-2)(k-3)}{4 !}, \cdots$,

$T_{n}=\frac{k(k-1) \cdots(k-n+1)}{n !}$

$$
\begin{gathered}
\sum_{i=1}^{n} i ! T_{i}=T_{1}+2 ! T_{2}+3 ! T_{3}+\cdots+n ! T_{n} \\
=k t+k(k-1)+k(k-1)(k-2) \\
+\cdots+k(k-1)(k-n+1) \\
\sum_{i=2}^{n} i(i-1) T_{i}=2 T_{2}+6 T_{3}+12 T_{4}+\cdots+n(n-1) T_{n} \\
=k(k-1)+k(k-1)(k-2)+\frac{1}{2} k(k-1)(k-2)(k-3)+\cdots \\
+\frac{1}{(n-2) !} k(k-1)(k-n+1)
\end{gathered}
$$




\section{Notion}

Let $R_{i}=R_{i}(P)$ represents the number of the $i$-secants (planes) through a point $P$ of a $(k, n)-\operatorname{arc} A$, in $P G(3, q)$ then the following equations are satisfied:

1) $\sum_{i=1}^{n} R_{i}=q^{2}+q+1$

$$
\sum_{i=2}^{n}(i-1) ! R_{i}
$$

2) $=(k-1)+(k-1)(k-2)+\cdots+(k-1)(k-2)(k-n-1)$

$$
=\sum_{i=1}^{n-1}(k-1)(k-i)
$$

\section{Proof}

1) $\sum_{i=1}^{n} R_{i}=R_{1}+R_{2}+\cdots+R_{n}, \sum_{i=1}^{n} R_{i}$ represents the sum of numbers of all the $i$-secants through a point $P$ of the $\operatorname{arc} A$, which is the number of the planes through $P$. Thus,

$$
\sum_{i=1}^{n} R_{i}=q^{2}+q+1
$$

2) $\sum_{i=2}^{n}(i-1) ! R_{i}=R_{2}+2 ! R_{3}+3 ! R_{4}+\cdots+(n-1) ! R_{n}$

$$
\begin{aligned}
& R_{2}=k-1, R_{3}=\left(\begin{array}{c}
k-1 \\
2
\end{array}\right), R_{4}=\left(\begin{array}{c}
k-1 \\
3
\end{array}\right), \cdots, R_{n}=\left(\begin{array}{c}
k-1 \\
n-1
\end{array}\right) \\
& R_{3}=\frac{(k-1) !}{2 !(k-3) !}, R_{4}=\frac{(k-1) !}{3 !(k-4) !}, \cdots, R_{n}=\frac{(k-1) !}{(n-1) !(k-n) !} \\
& R_{3}=\frac{(k-1)(k-2)}{2}, R_{4}=\frac{(k-1)(k-2)(k-3)}{3 !}, \cdots, R_{n}=\frac{(k-1) \cdots(k-(n-1))}{(n-1) !}
\end{aligned}
$$

$$
\begin{aligned}
\sum_{i=2}^{n}(i-1) ! R_{i}= & k-1+\frac{2 !(k-1)(k-2)}{2 !}+\frac{3 !(k-1)(k-2)(k-3)}{3 !}+\cdots \\
& +\frac{(n-1) !(k-1)(k-2) \cdots(k-(n-1))}{(n-1) !}
\end{aligned}
$$

$$
\begin{aligned}
= & (k-1)+(k-1)(k-2)+(k-1)(k-2)(k-3)+\cdots \\
& +(k-1)(k-2)(k-(n-1)) \\
= & \sum_{i=1}^{n-1}(k-1)(k-i)
\end{aligned}
$$

\section{Theorem}

Let $S_{i}=S_{i}(Q)$ represent the numbers of the $i$-secants (planes) of a $(k, n)$-arc $A$ through a point $Q$ in $P G(3, q)$ such that $Q$ not in $A$, then the following equations are satisfied:

1) $\sum_{i=0}^{n} S_{i}=q^{2}+q+1$ 
2) $\sum_{i=1}^{n} i S_{i}=k$

Proof

1) $\sum_{i=0}^{n} S_{i}$ represents the sum of the total numbers of all $i$-secants to $A$ through a point $Q$ not in $A$, which is equal to the number of all planes through the point $Q$. Thus $\sum_{i=0}^{n} S_{i}=q^{2}+q+1$.

2) $\sum_{i=1}^{n} i S_{i}=S_{1}+2 S_{2}+3 S_{3}+\cdots+n S_{n}$.

$S_{1}, S_{2}, \cdots, S_{n}$ represent the numbers of the $i$-secants of the arc $A$ through the point $Q$ not in $A$. $S_{1}$ is the number of the unisecants to $A$, each one passes through one point of $A . S_{2}$ is the number of the bisecants to $A$, each one passes through two points of $A$. $S_{3}$ is the number of the trisecants to $A$, each one passes through three points of $A$. Also, $S_{n}$ is the number of the $n$-secants to $A$, each one passes through $n$ points of $A$. Since the number of points of the $(k, n)$-arc $A$ is $k$, then $\sum_{i=1}^{n} i S_{i}=k$.

\section{Notion}

Let $C_{i}$ be the number of points of index $i$ in $S=P G(3, q)$ which are not on a complete $(k, n)$-arc $A$, then the constants $C_{i}$ of $A$ satisfy the following equations:
i) $\sum_{\alpha}^{\beta} C_{i}=q^{3}+q^{2}+q+1-k$
ii) $\sum_{\alpha}^{\beta} i C_{i}=\frac{k(k-1) \cdots(k-n+1)}{n !}\left(q^{2}+q+1-n\right)$

where $\alpha$ is the smallest $i$ for which $C_{i} \neq 0, \beta$ be the largest $i$ for which $C_{i} \neq 0$.

\section{Proof}

The equations express in different ways the cardinality of the following sets

i) $\{Q \mid Q \in S \backslash A\}$

ii) $\{(Q, \pi) / Q \in \pi \backslash A, \pi$ an $n$-secant of $A\}$

for in (i) $\sum_{\alpha}^{\beta} C_{i}$ represents all points in the space which are not in $A$, then $\sum_{\alpha}^{\beta} C_{i}=q^{3}+q^{2}+q+1-k$, and in (ii) $\sum_{\alpha}^{\beta} i C_{i}$ represents all points in the space not in $A$, which are on $n$-secants of $A$, that is, each $n$-secant contains $q^{2}+q+1-n$ points, and the number of the $n$-secants is $\left(\begin{array}{l}k \\ n\end{array}\right)$, then

$$
\begin{aligned}
\sum_{\alpha}^{\beta} i C_{i} & =\left(\begin{array}{l}
k \\
n
\end{array}\right)\left(q^{2}+q+1-n\right) \\
& =\frac{k(k-1) \cdots(k-n+1)}{n !}\left(q^{2}+q+1-n\right)
\end{aligned}
$$

\section{Theorem}

If $P$ is a point of a $(k, n)$-arc $A$ in $P G(3, q)$, which lies on an $m$-secant (plane) 
of $A$, then the planes through $P$ contains at most $(n-1) q(q+1)+m$ points of $A$.

\section{Proof}

If $P$ in $A$ lies on an $m$-secant (plane), then every other plane through $P$ contains at most $n-1$ points of A distinct from $P$. Hence the $q^{2}+q+1$ planes through $P$ contain at most $(n-1)\left(q^{2}+q\right)+m$ points of $A$.

\section{The Fundamental Theorem of Projective Geometry}

In this section, the characterization of algebraic varieties in $P G(3, q)$ is given as finite sets of points with certain combinatorial properties.

Definition: "Plane $\pi$ " [1]

A plane $\pi$ in $P G(3, p)$ is the set of all points $P\left(X_{1}, X_{2}, X_{3}, X_{4}\right)$ satisfying a linear equation $U_{1} X_{1}+U_{2} X_{2}+U_{3} X_{3}+U_{4} X_{4}=0$. This plane is denoted by $\pi\left[U_{1}, U_{2}, U_{3}, U_{4}\right]$. space which consists of points, lines and planes with the incidence relation between them [1].

\section{Notion [1]}

A projective 3-space $P G(3, k)$ over a field $K$ is a 3-dimensional projective $P G(3, k)$ satisfying the following axioms:

1) Any two distinct points are contained in a unique line.

2) Any three distinct non-collinear points, also any line and point not on the line are contained in a unique plane.

3) Any two distinct coplanar lines intersect in a unique point.

4) Any line not on a given plane intersects the plane in a uniquepoint.

5) Any two distinct planes intersect in a unique line.

A projective space $P G(3, p)$ over Galois field $G F(p)$, where $p=q^{m}$ For some prime number $q$ and some integer $m$, is a 3-dimensional projective space.

Any point in $P G(3, p)$ has the form of a quadruple $\left(X_{1}, X_{2}, X_{3}, X_{4}\right)$, where $X_{1}, X_{2}, X_{3}, X_{4}$ are elements in $G F(p)$ with the exception of the quadrable consisting of four zero elements. Two quadrable $\left(X_{1}, X_{2}, X_{3}, X_{4}\right)$ and $\left(y_{1}, y_{2}, y_{3}, y_{4}\right)$ represent the same point if there exists $\lambda$ in $G F(p) /\{0\}$ such that $\left(X_{1}, X_{2}, X_{3}, X_{4}\right)=\lambda\left(y_{1}, y_{2}, y_{3}, y_{4}\right)$. Similarly, any plane in $P G(3, p)$ has the form of a quadrable $\left[X_{1}, X_{2}, X_{3}, X_{4}\right]$, where $X_{1}, X_{2}, X_{3}, X_{4}$, are elements in $G F(p)$ with the exception of the quadrable consisting off our zero elements.

Two quadrable $\left[X_{1}, X_{2}, X_{3}, X_{4}\right]$ and $\left[y_{1}, y_{2}, y_{3}, y_{4}\right]$ represent the same plane if there exists $\lambda$ in $G F(p) \backslash\{0\}$ such that $\left[X_{1}, X_{2}, X_{3}, X_{4}\right]=\lambda\left[y_{1}, y_{2}, y_{3}, y_{4}\right]$.

Finally, a point $P\left(X_{1}, X_{2}, X_{3}, X_{4}\right)$ is incident with the plane $\pi\left[a_{1}, a_{2}, a_{3}, a_{4}\right]$ iff

$$
a_{1} X_{1}+a_{2} X_{2}+a_{3} X_{3}+a_{4} X_{4}=0 \text {. }
$$

Theorem [1] [19]

The points of $P G(3, p)$ have a unique forms which are $(1,0,0,0),(x, 1,0,0),(x, y, 1,0),(x, y, z, 1)$ for all $x, y, z$ in $G F(p)$.

There exists one point of the form $(1,0,0,0)$. 
There exists $p$ points of the form $(x, 1,0,0)$.

There exists $p^{2}$ points of the form $(x, y, 1,0)$.

There exists $p^{3}$ points of the form $(x, y, z, 1)$.

Theorem [19]

The planes of $P G(3, p)$ have a unique forms which are:

$[1,0,0,0],[x, 1,0,0],[x, y, 1,0],[x, y, z, 1]$ for all $x, y, z$ in $G F(p)$.

There exists one plane of the form $[1,0,0,0]$.

There exists $p$ planes of the form $[x, 1,0,0]$.

There exists $p^{2}$ planes of the form $[x, y, 1,0]$.

There exists $p^{3}$ planes of the form $[x, y, z, 1]$.

Notion [1]

In $P G(3, p)$ satisfies the following:

1) Every line contains exactly $p+1$ points and every point is on exactly $p+1$ lines.

2) Every plane contains exactly $p^{2}+p+1$ points (lines) and every point is on exactly $p^{2}+p+1$ planes.

3) There exist $p^{3}+p^{2}+p+1$ of points and there exists $p^{3}+p^{2}+p+1$ of planes.

4) Any two planes intersect in exactly $p+1$ points and any line is on exactly $P+1$ planes. So, any two points are on exactly $p+1$ planes.

Notion [1]

There exists $\left(p^{2}+1\right)\left(p^{2}+p+1\right)$ of lines in $P G(3, p)$.

Proof

In $P G(3, p)$, there exist $p^{3}+p^{2}+p+1$ planes, and each plane contains exactly $p^{2}+p+1$ lines, then the numbers of lines is equal to $\left(p^{3}+p^{2}+p+1\right)\left(p^{2}+p+1\right)$, but each line is on $p+1$ planes, then there exist exactly $\frac{\left(p^{3}+p^{2}+p+1\right)\left(p^{2}+p+1\right)}{p+1}=\left(p^{2}+1\right)\left(p^{2}+p+1\right)$ lines in $P G(3, p)$.

Qualifier [1] [19]

A ( $k$, $)$-span,,$\geq 1$ is a set of $k$ spaces $\pi_{q}$ no two of which intersect.

Definition [1]

A maximum $(k, 0)$-span is a set of $k$ spaces $\pi_{\text {l }}$ which are every points of $P G(3, p)$ lies in exactly one line of the, and every two lines of $\pi_{\mathfrak{q}}$ are disjoint

Qualifier [1] [19]

Every maximum $(k$, , $)$-span is a spread.

\section{The Projective Space and the $(k, \eta)$-Span in $P G(3,7)$}

\subsection{The Projective Space in $P G(3,7)$}

$P G(3,7)$ contains 400 points and 400 planes such that each point is on 57 planes and every plane contains 57 points, any line contains 8 points and it is the intersection of 8 planes, all the points, planes and lines of $P G(3,7)$ are given in Table 1 and Table 2. 
Table 1. Points and plane of $P G(3,7)$.

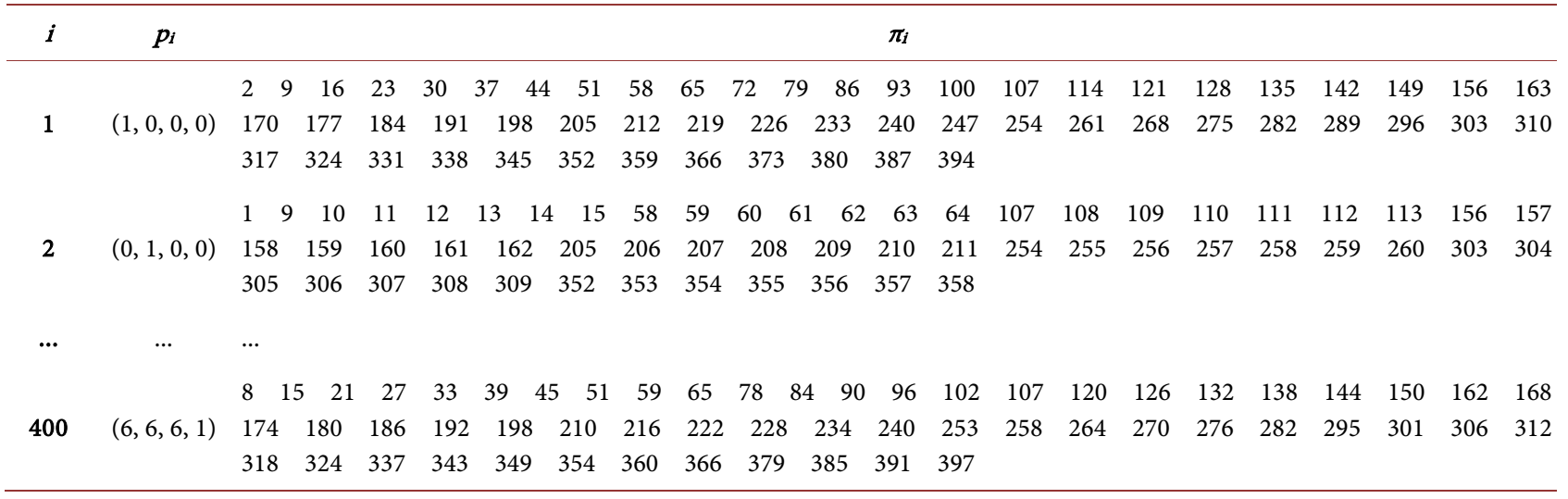

Table 2. Plane and lines of $P G(3,7)$.

$\begin{array}{lllllllllllllllllllllllllllllllllllllll}1 & 2 & 2 & 2 & 2 & 2 & 2 & 2 & 2 & 9 & 9 & 9 & 9 & 9 & 9 & 9 & 16 & 16 & 16 & 16 & 16 & 16 & 16 & 23 & 23 & 23 & 23 & 23 & 23 & 23 & 30 & 30 & 30 & 30 & 30 & 30 & 30\end{array}$

$\begin{array}{lllllllllllllllllllllllllllllllllllll}9 & 58 & 107 & 156 & 205 & 254 & 303 & 352 & 58 & 65 & 72 & 79 & 86 & 93 & 100 & 58 & 65 & 72 & 79 & 86 & 93 & 100 & 58 & 65 & 72 & 79 & 86 & 93 & 100 & 58 & 65 & 72 & 79 & 86 & 93 & 100\end{array}$

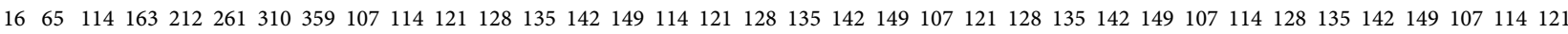

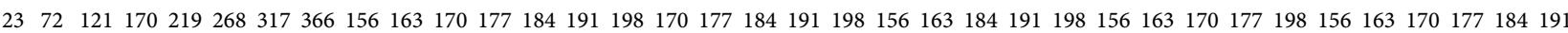
$30 \quad 79128177226275324373205212219226233240247226233240247205212219247205212219226233240219226233240247205212$

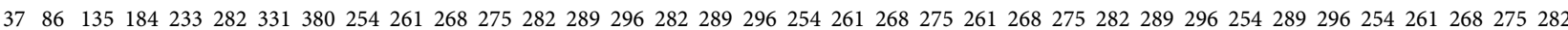
$4493 \quad 142191240289338387303310317324331338345338345303 \quad 310317324331324331338345303310317310317324331338345303$

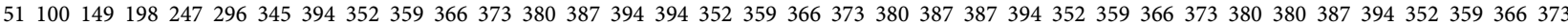

\begin{tabular}{|c|c|c|c|c|c|c|c|c|c|c|c|c|c|c|c|c|c|c|c|c|}
\hline 37 & 37 & 37 & 37 & 37 & 37 & 37 & 44 & 44 & 44 & 44 & 44 & 44 & 44 & 51 & 51 & 51 & 51 & 51 & 51 & 51 \\
\hline 58 & 65 & 72 & 79 & 86 & 93 & 100 & 58 & 65 & 72 & 79 & 86 & 93 & 100 & 58 & 65 & 72 & 79 & 86 & 93 & 100 \\
\hline 135 & 142 & 149 & 107 & 114 & 121 & 128 & 142 & 149 & 107 & 114 & 121 & 128 & 135 & 149 & 107 & 114 & 121 & 128 & 135 & 142 \\
\hline 163 & 170 & 177 & 184 & 191 & 198 & 156 & 177 & 184 & 191 & 198 & 156 & 163 & 170 & 191 & 198 & 156 & 163 & 170 & 177 & 184 \\
\hline 240 & 247 & 205 & 212 & 219 & 226 & 233 & 212 & 219 & 226 & 233 & 240 & 247 & 205 & 233 & 240 & 247 & 205 & 212 & 219 & 226 \\
\hline 268 & 275 & 282 & 289 & 296 & 254 & 261 & 296 & 254 & 261 & 268 & 275 & 282 & 289 & 275 & 282 & 289 & 296 & 254 & 261 & 268 \\
\hline 345 & 303 & 310 & 317 & 324 & 331 & 338 & 331 & 338 & 345 & 303 & 310 & 317 & 324 & 317 & 324 & 331 & 338 & 345 & 303 & 310 \\
\hline 373 & 380 & 387 & 394 & 352 & 359 & 366 & 366 & 373 & 380 & 387 & 394 & 352 & 359 & 359 & 366 & 373 & 380 & 387 & 394 & 352 \\
\hline
\end{tabular}

$\begin{array}{llllllllllllllllllllllllllllllllllllllll}1 & 1 & 1 & 1 & 1 & 1 & 1 & 1 & 9 & 9 & 9 & 9 & 9 & 9 & 9 & 10 & 10 & 10 & 10 & 10 & 10 & 10 & 11 & 11 & 11 & 11 & 11 & 11 & 11 & 12 & 12 & 12 & 12 & 12 & 12 & 12\end{array}$

$\begin{array}{llllllllllllllllllllllllllllllllllll}9 & 58 & 107 & 156 & 205 & 254 & 303 & 352 & 58 & 59 & 60 & 61 & 62 & 63 & 64 & 58 & 59 & 60 & 61 & 62 & 63 & 64 & 58 & 59 & 60 & 61 & 62 & 63 & 64 & 58 & 59 & 60 & 61 & 62 & 63 & 64\end{array}$

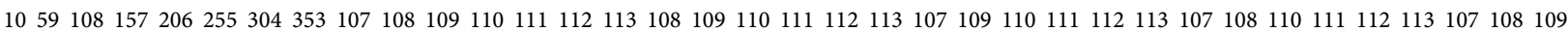

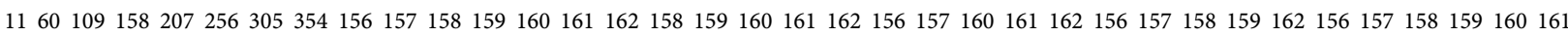
1261110159208257306355205206207208209210211208209210211205206207211205206207208209210207208209210211205206 1362111160209258307356254255256257258259260258259260254255256257255256257258259260254259260254255256257258 1463112161210259308357303304305306307308309308309303304305306307306307308309303304305304305306307308309303 1564113162211260309358352353354355356357358358352353354355356357357358352353354355356356357358352353354355 


\begin{tabular}{|c|c|c|c|c|c|c|c|c|c|c|c|c|c|c|c|c|c|c|c|c|}
\hline 13 & 13 & 13 & 13 & 13 & 13 & 13 & 14 & 14 & 14 & 14 & 14 & 14 & 14 & 15 & 15 & 15 & 15 & 15 & 15 & 15 \\
\hline 58 & 59 & 60 & 61 & 62 & 63 & 64 & 58 & 59 & 60 & 61 & 62 & 63 & 64 & 58 & 59 & 60 & 61 & 62 & 63 & 64 \\
\hline 111 & 112 & 113 & 107 & 108 & 109 & 110 & 112 & 113 & 107 & 108 & 109 & 110 & 111 & 113 & 107 & 108 & 109 & 110 & 111 & 112 \\
\hline 157 & 158 & 159 & 160 & 161 & 162 & 156 & 159 & 160 & 161 & 162 & 156 & 157 & 158 & 161 & 162 & 156 & 157 & 158 & 159 & 160 \\
\hline 256 & 257 & 258 & 259 & 260 & 254 & 255 & 260 & 254 & 255 & 256 & 257 & 258 & 259 & 257 & 258 & 259 & 260 & 254 & 255 & 256 \\
\hline 309 & 303 & 304 & 305 & 306 & 307 & 308 & 307 & 308 & 309 & 303 & 304 & 305 & 306 & 305 & 306 & 307 & 308 & 309 & 303 & 304 \\
\hline 355 & 356 & 357 & 358 & 352 & 353 & 354 & 354 & 355 & 356 & 357 & 358 & 352 & 353 & 353 & 354 & 355 & 356 & 357 & 358 & 352 \\
\hline
\end{tabular}

$\begin{array}{lllllllllllllllllllllllllllllllllllll}8 & 8 & 8 & 8 & 8 & 8 & 8 & 8 & 15 & 15 & 15 & 15 & 15 & 15 & 15 & 21 & 21 & 21 & 21 & 21 & 21 & 21 & 27 & 27 & 27 & 27 & 27 & 27 & 27 & 33 & 33 & 33 & 33 & 33 & 33 & 33\end{array}$

$\begin{array}{llllllllllllllllllllllllllllllllllll}15 & 59 & 107 & 162 & 210 & 258 & 306 & 354 & 59 & 65 & 78 & 84 & 90 & 96 & 102 & 59 & 65 & 78 & 84 & 90 & 96 & 102 & 59 & 65 & 78 & 84 & 90 & 96 & 102 & 59 & 65 & 78 & 84 & 90 & 96 & 102\end{array}$

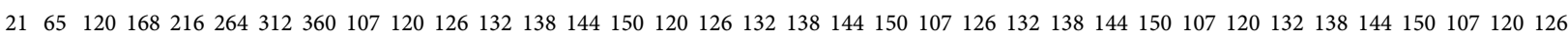

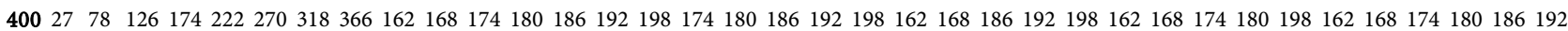

3384132180228276324379210216222228234240253228234240253210216222253210216222228234240222228234240253210216 3990138186234282337385258264270276282295301282295301258264270276264270276282295301258295301258264270276282 4596144192240295343391306312318324337343349343349306312318324337324337343349306312318312318324337343349306 51102150198253301349397354360366379385391397397354360366379385391391397354360366379385385391397354360366379

\begin{tabular}{|c|c|c|c|c|c|c|c|c|c|c|c|c|c|c|c|c|c|c|c|c|}
\hline 39 & 39 & 39 & 39 & 39 & 39 & 39 & 45 & 45 & 45 & 45 & 45 & 45 & 45 & 51 & 51 & 51 & 51 & 51 & 51 & 51 \\
\hline 59 & 65 & 78 & 84 & 90 & 96 & 102 & 59 & 65 & 78 & 84 & 90 & 96 & 102 & 59 & 65 & 78 & 84 & 90 & 96 & 102 \\
\hline 168 & 174 & 180 & 186 & 192 & 198 & 162 & 180 & 186 & 192 & 198 & 162 & 168 & 174 & 192 & 198 & 162 & 168 & 174 & 180 & 186 \\
\hline 240 & 253 & 210 & 216 & 222 & 228 & 234 & 216 & 222 & 228 & 234 & 240 & 253 & 210 & 234 & 240 & 253 & 210 & 216 & 222 & 228 \\
\hline 270 & 276 & 282 & 295 & 301 & 258 & 264 & 301 & 258 & 264 & 270 & 276 & 282 & 295 & 276 & 282 & 295 & 301 & 258 & 264 & 270 \\
\hline 349 & 306 & 312 & 318 & 324 & 337 & 343 & 337 & 343 & 349 & 306 & 312 & 318 & 324 & 318 & 324 & 337 & 343 & 349 & 306 & 312 \\
\hline 379 & 385 & 391 & 397 & 354 & 360 & 366 & 366 & 379 & 385 & 391 & 397 & 354 & 360 & 360 & 366 & 379 & 385 & 391 & 397 & 354 \\
\hline
\end{tabular}

Table 3. Spread in $P G(3,7)$.

\begin{tabular}{|c|c|c|c|c|c|c|c|c|c|}
\hline \multirow{2}{*}{$\begin{array}{l}t_{i} \\
\xi \xi\end{array}$} & \multicolumn{8}{|c|}{$9 i$} & \multirow{2}{*}{$\frac{\left(k_{i}, \mathfrak{Q}\right) \text {-span }}{(1, \text { ) } \text {-span }}$} \\
\hline & 1 & 2 & 3 & 4 & 5 & 6 & 7 & 8 & \\
\hline$v$ & 9 & 58 & 107 & 156 & 205 & 254 & 303 & 352 & $(2,0)$-span \\
\hline$\mu$ & 10 & 65 & 115 & 165 & 215 & 265 & 315 & 365 & $(3,0)$-span \\
\hline$\theta$ & 11 & 72 & 123 & 174 & 225 & 269 & 320 & 371 & $(4$, ) -span \\
\hline$\eta$ & 12 & 79 & 131 & 183 & 228 & 280 & 325 & 377 & $(5,0)$-span \\
\hline$\zeta$ & 13 & 86 & 139 & 185 & 238 & 284 & 337 & 383 & $(6,9)$-span \\
\hline $\boldsymbol{\varepsilon}$ & 14 & 93 & 147 & 194 & 241 & 295 & 342 & 389 & $(7,0)$-span \\
\hline$\delta$ & 15 & 100 & 155 & 203 & 251 & 299 & 347 & 395 & $(8,0)$-span \\
\hline$\gamma$ & 16 & 61 & 117 & 173 & 229 & 285 & 341 & 397 & $(9,0)$-span \\
\hline$\beta$ & 17 & 68 & 125 & 182 & 239 & 289 & 346 & 354 & $(10, \emptyset)$-span \\
\hline
\end{tabular}




\section{Continued}

\begin{tabular}{|c|c|c|c|c|c|c|c|c|c|}
\hline$\alpha$ & 18 & 75 & 133 & 184 & 242 & 300 & 309 & 360 & $(11$, Q)-span \\
\hline$\vartheta$ & 19 & 82 & 141 & 193 & 252 & 255 & 314 & 366 & $(12,9)$-span \\
\hline$B$ & 20 & 89 & 142 & 202 & 206 & 266 & 319 & 379 & $(13$, , $)$-span \\
\hline$\dot{\omega}$ & 21 & 96 & 150 & 162 & 216 & 270 & 324 & 385 & $(14,0)$-span \\
\hline$\dot{v}$ & 22 & 103 & 109 & 164 & 219 & 281 & 336 & 391 & $(15, \mathrm{Q})$-span \\
\hline ó & 23 & 64 & 127 & 190 & 253 & 267 & 330 & 393 & $(16,9)$-span \\
\hline$\ddot{v}$ & 24 & 71 & 128 & 192 & 207 & 271 & 335 & 399 & $(17,9)$-span \\
\hline$\ddot{\imath}$ & 25 & 78 & 136 & 201 & 217 & 275 & 340 & 356 & $(18,0)$-span \\
\hline$\omega$ & 26 & 85 & 144 & 161 & 220 & 286 & 345 & 362 & $(19$, Q)-span \\
\hline$\psi$ & 27 & 92 & 152 & 163 & 230 & 290 & 308 & 368 & $(20,0)$-span \\
\hline$\sigma$ & 28 & 99 & 111 & 172 & 233 & 301 & 313 & 374 & $(21, \mathrm{Q})$-span \\
\hline$\varsigma$ & 29 & 106 & 119 & 181 & 243 & 256 & 318 & 380 & $(22, \mathrm{Q})$-span \\
\hline$\rightarrow$ & 30 & 60 & 130 & 200 & 221 & 291 & 312 & 382 & $(23$, Q)-span \\
\hline T & 31 & 67 & 138 & 160 & 231 & 302 & 317 & 388 & $(24$, Q) -span \\
\hline 4 & 32 & 74 & 146 & 169 & 234 & 257 & 329 & 394 & $(25$, , )-span \\
\hline$F$ & 33 & 81 & 154 & 171 & 244 & 261 & 334 & 358 & $(26, \mathrm{Q})$-span \\
\hline$Q$ & 34 & 88 & 113 & 180 & 247 & 272 & 339 & 364 & $(27$, Q)-span \\
\hline$\omega$ & 35 & 95 & 114 & 189 & 208 & 276 & 351 & 370 & $(28,0)$-span \\
\hline @ & 36 & 102 & 122 & 191 & 218 & 287 & 307 & 376 & $(29$, Q) -span \\
\hline 6 & 37 & 63 & 140 & 168 & 245 & 273 & 350 & 378 & $(30, \mathrm{Q})$-span \\
\hline$x$ & 38 & 70 & 148 & 170 & 248 & 277 & 306 & 384 & $(31, \mathrm{Q})$-span \\
\hline b & 39 & 77 & 149 & 179 & 209 & 288 & 311 & 390 & $(32,9)$-span \\
\hline Шు & 40 & 84 & 108 & 188 & 212 & 292 & 323 & 396 & $(33, \mathrm{Q})$-span \\
\hline$\epsilon$ & 41 & 91 & 116 & 197 & 222 & 296 & 328 & 353 & $(34, \mathrm{Q})$-span \\
\hline Б & 42 & 98 & 124 & 199 & 232 & 258 & 333 & 359 & $(35$, Q)-span \\
\hline џ & 43 & 105 & 132 & 159 & 235 & 262 & 338 & 372 & $(36,0)$-span \\
\hline y̆ & 44 & 59 & 143 & 178 & 213 & 297 & 332 & 367 & $(37,0)$-span \\
\hline ѝ & 45 & 66 & 151 & 187 & 223 & 259 & 344 & 373 & $(38,9)$-span \\
\hline $\mathbf{h}$ & 46 & 73 & 110 & 196 & 226 & 263 & 349 & 386 & $(39,9)$-span \\
\hline њ & 47 & 80 & 118 & 198 & 236 & 274 & 305 & 392 & $(40$, ()-span \\
\hline Љ & 48 & 87 & 126 & 158 & 246 & 278 & 310 & 398 & $(41, \mathrm{Q})$-span \\
\hline ' & 49 & 94 & 134 & 167 & 249 & 282 & 322 & 355 & $(42, \mathrm{Q})$-span \\
\hline Л & 50 & 101 & 135 & 176 & 210 & 293 & 327 & 361 & $(43$, Q) -span \\
\hline щ & 51 & 62 & 153 & 195 & 237 & 279 & 321 & 363 & $(44$, Q)-span \\
\hline Ю & 52 & 69 & 112 & 204 & 240 & 283 & 326 & 369 & $(45$, , $)$-span \\
\hline Ы & 53 & 76 & 120 & 157 & 250 & 294 & 331 & 375 & $(46$, Q)-span \\
\hline ж & 54 & 83 & 121 & 166 & 211 & 298 & 343 & 381 & $(47,9)$-span \\
\hline ц & 55 & 90 & 129 & 175 & 214 & 260 & 348 & 387 & $(48$, Q)-span \\
\hline व & 56 & 97 & 137 & 177 & 224 & 264 & 304 & 400 & $(49,9)$-span \\
\hline GD & 57 & 104 & 145 & 186 & 227 & 268 & 316 & 357 & $(50$, $)$-span \\
\hline
\end{tabular}




\subsection{The $(k$, , $)$-Span in $P G(3, p)$}

In Table 3 any two non-intersecting lines can be taken in $P G(3,7)$.

In Table 3 any elements of the set $t_{i}=\{\xi, v, \mu, \cdots, G\}$ except the first element can be representing by union of below set and non-intersecting of them.

Finally, the line $G=\{57,104,145,186,227,268,316,357\}$ cannot intersect any line of the set $\left(\mathrm{t}_{i}\right)$ and $(\mathrm{G})$ is (50,0)-span, which is the maximum (k, )-span of $P G(3,7)$ can be obtained. Thus $G$ is called a Spread of fifty lines of $P G(3,7)$ which partitions $P G(3,7)$; that every point of $P G(3,7)$ lies in exactly one line of $t_{i}$ and every line are disjoint. From the above results the number of the planes in the projective space

$P G(3,7)$ are 400 planes and each plane contains 57 lines, therefore the total number of the lines in $P G(3,7)$ are 22,800 . We found that the number of the lines do not intersect with some of them are fifty lines, these lines contains the whole points of the projective space $P G(3,7)$, and called him a (50, )-span, i.e.

$$
\left(50, \text { )-span }=\left\{q_{1}, q_{2}, \cdots, q_{50}\right\}=P G(3,7)=\{1,2,3, \cdots, 400\}\right.
$$

Moreover, we found that a (50,१)-span is a maximum complete $(k, \mathrm{Q})$-span in $P G(3,7)$.

\section{Conclusions}

1) Complete Arcs and Surfaces are constructed in $P G(3, q)$ over Galois field $G F(q), q=7$ by two methods and some theorems are proved on the $(k, n)$-arc of $P G(3, q)$ and on a 3-dimensional projective space.

2) We prove, the number of spread in projective space $P G(3, p)$ where $p$ is prime, and $P \geq 2$ is $p^{2}+1$.

\section{Proof}

In $P G(3, p)$, there are $p^{3}+p^{2}+p+1$ planes, but each line is on $p+1$ planes; then there is exactly $\frac{p^{3}+p^{2}+p+1}{p+1}=p^{2}+1$ spread in $P G(3, p)$.

\section{Conflicts of Interest}

The authors declare no conflicts of interest regarding the publication of this paper.

\section{References}

[1] Hirschfeld, J.W.P. (1979) Projective Geometries over Finite Fields. Oxford Clarendon Press, Oxford, Oxford University Press, New York. https://www.researchgate.net/publication/336672313

[2] Kareem, F.F. and Kadhum, S.J. (2013) A (k,l) Span in Three Dimensional Projective Space PG $(3, \mathrm{p})$ over Galois Field Where $\mathrm{p}=4$. Journal of the College of Basic Education, 19, 659-672. https://www.iasj.net/iasj?func=article\&aId=149299

[3] AL-Mukhtar, A.S. (2008) Complete Arcs and Surfaces in Three-Dimensional Projective Space over Galois Field. PHD Thesis, University of Technology, Baghdad. https://www.iasj.net/iasj?func=fulltext\&aId $=44179$ 
[4] Ibrahim, H.S. and Kasm, N.Y. (2019) The Possibility of Applying Rumen Research at the Projective Plane PG(2,17). Modern Applied Science, 13, 150. https://doi.org/10.5539/mas.v13n8p150

[5] Ibrahim, H.S. and Kasm, N.Y. (2019) The Optimal Size of $\{b, t\}$-Blocking Set When $t$ = 3, 4 by Intersection the Tangents in PG(2,q). Modern Applied Science, 13, 15. https://doi.org/10.5539/mas.v13n7p15

[6] Kasm, N.Y. and Hamad, A.Z. (2019) Applications of Algebraic Geometry in Cryptography. Modern Applied Science, 13, 130. https://doi.org/10.5539/mas.v13n5p130

[7] Yahya, N.Y.K. (2018) A Geometric Construction of Complete (k,r)-arc in PG(2,7) and the Related Projective [n,3,d]7 Codes. AL-Rafidain Journal of Computer Sciences and Mathematics, 12, 24-40. https://doi.org/10.33899/csmj.2018.163568

[8] Yahya, N.Y.K. (2014) A Non PGL $(3, q)$ k-Arcs in the Projective Plane of Order 37. Tikrit Journal of Pure Science, 19, 135-145.

[9] Yahya, N.Y.K. (2012) Existence of Minimal Blocking Sets of Size 31 in the Projective Plane PG(2,17). Journal of University of Babylon/Pure and Applied Sciences, 20, 1138-1146.

[10] Yahya, N.Y.K. (2014) The Use of 7-Blocking Sets in Galois Geometries. Journal of University of Babylon/ Pure and Applied Sciences, 22, 1229-1235.

[11] Yahya, N.Y.K. and Salim, M.N. (2018) The Geometric Approach to Existences Linear $[\mathrm{n}, \mathrm{k}, \mathrm{d}] 13$ Code. International Journal of Enhanced Research in Science, Technology and Engineering.

https://www.academia.edu/39969897/The Geometric Approach to Existence Lin ear $\mathrm{n} \mathrm{k} \mathrm{d} 13$ Codes

[12] Yahya, N.Y.K. and Salim, M.N. (2019) New Geometric Methods for Prove Existence Three-Dimensional Linear [97,3,87]11 and [143,3,131]13 Codes. Journal of Education and Science, 28, 312-333. https://doi.org/10.33899/edusj.2019.161057

[13] Yahya, N.Y.K. and Salim, M.N. (2019) 17 New Existences Linear [n,3,d]19 Codes by Geometric Structure Method in PG(2,19). AL-Rafidain Journal of Computer Sciences and Mathematics, 13, 61-86. https://doi.org/10.33899/csmj.2020.163503

[14] Yahya, N.Y.K. and Hamad, A.Z. (2019) A Geometric Construction of a $(56,2)$ Blocking Set in PG(2,19) and on Three Dimensional Linear [325,3,307]19 Griesmer Code. University of Mosul, Mosul-Iraq. AL-Rafidain Journal of Computer Sciences and Mathematics, 13, 13-25. https://www.researchgate.net/publication/332718520 https://doi.org/10.33899/csmj.2020.163511

[15] Sulaimaan, A.E.M. and Kasm, N.Y. (2019) Linear Code Originates from Complete Arc by Engineering Building Methods. Applied Mathematical Science, 13, 1013-1020. https://doi.org/10.12988/ams.2019.99132

[16] Sulaimaan, A.E.M. and Kasm, N.Y. (2019) Linear Code Related $\Omega$-Blocking Sets. Applied Mathematical Science, 13, 1033-1040.

https://doi.org/10.12988/ams.2019.99133

[17] Sulaimaan, A.E.M. and Kasm, N.Y. (2019) New Structural Engineering Construction Method by Remove q Points in PG(2,q). Applied Mathematical Science, 13, 1048-1041.

https://www.researchgate.net/publication/336613294 New Structural Engineering Construction Methods by Remove q Points in PG2q https://doi.org/10.12988/ams.2019.99134

[18] Faraj, M.G. and Kasm, N.Y. (2019) Reverse Building of Complete (k,r)-Arcs in PG(2,q). Open Access Library Journal, 6, e5900.

https://www.researchgate.net/publication/338120917_Reverse_Building_of Comple 
te $\mathrm{kr}$-Arcs in PG2q

https://doi.org/10.4236/oalib.1105900

[19] Glynn, D.G. (2010) Theorems of Points and Planes in Three-Dimensional Projective Space. Journal of the Australian Mathematical Society, 88, 75-92.

https://www.academia.edu/25562079/Theorems of Points and Planes in Three-

Dimensional Projective Space

https://doi.org/10.1017/S1446788708080981

[20] Hirschfeld, J.W.P. (1998) Projective Geometries over Finite Fields. Second Edition, Oxford Clarendon Press, Oxford, Oxford University Press, New York.

https://global.oup.com/academic/product/projective-geometries-over-finite-fields-9 $\underline{780198502951 ? \mathrm{cc}=\mathrm{dk} \& \text { lang }=\mathrm{en} \&}$ 